\title{
Errores en discurso escrito en inglés por futuros profesores de inglés como lengua extranjera*
}

\author{
Eladio Donoso** \\ Romina Gómez***
}

\begin{abstract}
Resumen
Los futuros profesores de inglés en Chile deben, durante su formación, alcanzar un alto dominio de competencia comunicativa. Este artículo da cuenta de errores gramaticales cometidos en discurso escrito por futuros profesores de inglés, en contexto de inglés como lengua extranjera, de distintos niveles de su plan curricular. Los resultados señalan que existen diferencias en el número y tipo de error cometidos por los sujetos de los distintos niveles, mostrando una mayor diferencia entre los sujetos de primer y cuarto año. Esto permite describir su estado de interlengua.
\end{abstract}

Palabras clave: formación de profesores de inglés, error gramatical, interlengua, lengua materna, segunda lengua, lengua extranjera.

\section{Grammatical Errors in English Written Discourse Committed by EFL Teacher Trainees}

\begin{abstract}
Future teachers of English in Chile must, during their training, achieve a high level of communicative competence. This article gives an account of grammatical errors committed in written discourse by future teachers of English, in English as a foreign language context, of different levels of their curriculum. Results indicate that there are differences in the number and type of error committed by the participants of the different levels, showing a greater difference between the participants of first and fourth year. This permits to describe their interlanguage stage.
\end{abstract}

Keywords: EFL teacher training, Grammatical error, Interlanguage, First language, Second language, Foreign language.

Recibido: 11/01/2018

Aceptado: 01/o6/2018

\footnotetext{
* $\quad$ Este artículo es parte del Proyecto Semilla UCN2017 Creencias y Realidades de Futuros y Actuales Profesores de Inglés como Lengua Extranjera de la Vicerrectoría de Investigación y Desarrollo Tecnológico VRIDT de la Universidad Católica del Norte. Se agradece profundamente a Valentina Carrizo, Verónica Muñoz y Lorena Sánchez por su significativa colaboración en el equipo de investigación.

** Chileno. Doctor en Lingüística de la Pontificia Universidad Católica de Valparaíso. Escuela de Inglés, Universidad Católica del Norte, Antofagasta, Chile. edonoso@ucn.cl

*** Chilena. Magister en Educación de la Universidad La República. Jefa de Innovación Curricular de Centro de Innovación Metodológica y Tecnológica CIMET Universidad Católica del Norte, Antofagasta, Chile.rgomezo2@ucn.cl
} 


\section{Introducción}

La lengua inglesa se ha convertido en el idioma universal en los ámbitos de la economía, la cultura, la ciencia, el turismo, la tecnología y la comunicación en general. Es por ello que se ha transformado en un eje principal en la política educacional del Estado de Chile, el cual ha impulsado decididamente iniciativas que apuntan al desarrollo de las competencias comunicativas de dicha lengua. Esto hace más de una década. Entre estas iniciativas se encuentran, por ejemplo, el programa Inglés Abre Puertas, Programa Mecesup para mejoramiento de los programas de pedagogía en inglés y el programa de Semestre en el Extranjero para estudiantes de pedagogía en inglés (Mineduc, s.ñf.).

No obstante lo anterior, los resultados que se han obtenido en las mediciones hechas sobre el nivel de inglés de los profesores y estudiantes del sistema nacional chileno, tales como el SIMCE y otros, distan mucho de lo esperado por los estándares establecidos por el Mineduc (Barahona, 2016). Ante este panorama, es relevante indagar cuáles podrían ser las razones de estos bajos resultados. Es así que la investigación que se informa en este artículo se centra particularmente en los profesores, específicamente aquellos en formación, como uno de los múltiples factores y agentes involucrados en el proceso de enseñanza-aprendizaje del inglés como lengua extranjera en Chile.

Los futuros profesores de inglés como lengua extranjera (ILE) adscritos a programas de pedagogía en inglés en Chile son aprendientes de dicha lengua y como tales deberían alcanzar un alto dominio de competencia comunicativa, de tal manera que sean buenos modelos para sus futuros estudiantes. Como aprendientes de una lengua extranjera (LE), también llamada genéricamente lengua dos (L2) o lengua meta (LM), se caracterizan por el desarrollo de un sistema que se denomina interlengua (Selinker, 1972), que es un tipo de lengua que resulta de la interacción, transferencia o influencia entre el sistema la lengua materna o lengua uno (L1), de la LM, o bien, producto del desarrollo mismo de la competencia.

De esta forma, este artículo da cuenta de los errores gramaticales de interlengua que se observaron a través de los errores cometidos en la producción de discurso escrito por parte específicamente de una muestra de población de estos aprendientes, quienes serán futuros profesores de inglés como lengua extranjera que cursan los niveles de primero, segundo, tercero y cuarto de su carrera de una universidad chilena. 
El fenómeno de interlengua alcanza aún más relieve puesto que una posible fosilización de los errores de por parte del futuro profesor de inglés no solo lo afectará en su actuación comunicativa, sino que también a sus futuros estudiantes, en tanto que esos errores podrían ser traspasados a ellos, los estudiantes.

Son numerosos los estudios realizados en los últimos años sobre errores cometidos por aprendientes de una L2 y que reflejen su estado de interlengua (Bourguignon, 2008-2009; Sánchez Jiménez, 2010; Yuanrong \& Mingcai, 2010; Krishnamurthy, Kangira, Tjiramanga \& Beukes, 2011; Ahour \& Mukundan, 2012; Bailini, 2012; Liu, 2012; Mutema \& Mariko, 2012; Khansir \& Shahhoseiny, 2013; Zheng \& Park, 2013; Campillos Llanos, 2014; Doquin de Saint Preux \& Sáez Garcerán, 2014; Jagusztyn, 2014; Li, 2015; Murad \& Khalil, 2015; Zhan, 2015; Kurniawan, 2016). Sin embargo, la literatura especializada no da cuenta de estudios que comprendan una muestra de población compuesta por futuros profesores de lenguas extranjeras, ni tampoco que evidencien diferencias dependiendo del nivel que cursen dichos sujetos. De este modo, en este artículo se responde a la pregunta respecto a qué tipos de errores gramaticales cometen con mayor frecuencia profesores de inglés en formación dependiendo del nivel que cursen y que, a su vez, den cuenta de su interlengua.

En este contexto, el presente artículo presenta primero algunos referentes teóricos. Luego se informa sobre la metodología empleada en el estudio para finalmente mostrar los resultados y discusión, así como las conclusiones.

\section{Referentes teóricos}

Durante varios años, el término interlengua fue conocido como idiosyncratic dialect (Corder, 1967) y approximative system (Nemser, 1971). Sin embargo, fue el investigador Larry Selinker quien, en el año 1972, acuñó el término interlengua para referirse a las tentativas de producción significativa de aprendientes adultos de una lengua extranjera, es decir, cuando intentan expresar, en un idioma que aún no dominan, conceptos que ya conocen en su propia lengua. Uno de los factores más destacados por los investigadores sobre interlengua es que se caracteriza por aparecer en un grupo etario que ya ha pasado la pubertad, siendo así denominado como un sistema propio de los aprendientes adultos (Tarone, 2006). 
Esta lengua propia del aprendiente es una aproximación a la lengua meta que se alcanza a partir de elementos que provienen de su propia lengua, así como también de la lengua que se aprende y del desarrollo mismo que compromete el proceso de aprendizaje, los cuales intervienen e interfieren, lo que lo lleva a cometer errores. En este ámbito, Fernández (1997) señala que el análisis de errores corresponde a la metodología de investigación de la interlengua.

Pues bien, los errores ayudan a investigadores a realizar una categorización del nivel en que los aprendientes se encuentran, es decir, su cercanía a la lengua meta. Insistimos en que este proceso ocurre principalmente en aprendientes adultos, ya que difícilmente se puede encontrar en niños. Esto significa que los aprendientes adultos de una L2 nunca llegan a poseer un dominio íntegro de la lengua meta y dichas condiciones se hacen notar principalmente en los momentos de ansiedad, estrés, inatención o en presencia de nuevas reglas lingüísticas (Corder, 1967, 1981; Krashen, 1981; Selinker, 1972; Tarone, 2006, 2014).

En el proceso de aprender una L2, los errores son comunes y esperados. Al referirnos al error, es importante mencionar que este término se asocia a dos acepciones en lengua inglesa, lo que nos parece importante destacar: error y mistake. En su libro The significance of learner's error, Corder (1967) propone la diferencia entre estos dos términos, definiendo error como errores de performance que proveen evidencia del sistema de lengua usado por un aprendiente en un punto particular de su aprendizaje. En cambio mistake es definido como un lapsus y no es relevante para el proceso de aprendizaje de un segundo idioma. Por su parte, Ellis (2012) define mistake como una inatención que se manifiesta en el discurso del hablante y que es notada por este y corregida oportunamente.

El estudio de errores puede dar luces sobre el fenómeno denominado fosilización. Selinker (1972) profundiza en el concepto de la fosilización de la interlengua, describiendo este fenómeno lingüístico como un sistema que posee ítems, reglas y subsistemas, los cuales mantienen los aprendientes de una lengua, por lo tanto, su interlengua se estabiliza. Esto sucederá sin importar las explicaciones e instrucción que reciba en lo sucesivo el aprendiente.

De esta forma, el análisis de los errores nos entrega un acceso a información observable de la cual se pueden hacer inferencias sobre la 
interlengua de los aprendientes. De acuerdo a diferentes autores (Corder, 1981; James, 1998; Ellis, 2012; entre otros), los errores cometidos dentro del contexto del aprendizaje de una L2 son piezas clave que delatan el nivel en que los estudiantes se encuentran, es decir, la competencia que han alcanzado hasta un momento determinado.

Del mismo modo, el análisis de errores ayuda al proceso de enseñanza y aprendizaje para que así el profesor aplique metodologías que sean propicias a las necesidades de los estudiantes (Heydari y Bagheri, 2012). En esta línea, Johnson (2001) hace la siguiente analogía para explicar la importancia de observar los errores: "Errors hold vital clues about the process of EFL learning, rather like the pain that may tell the doctor more than all the parts that do not hurt. All shows that teachers and learners will benefit from various forms of feedback on the errors, so it is necessary to focus on language learners' errors." (p. 59).

Esto sugiere que los errores son realmente importantes puesto que pueden, como ya se ha dicho, aportar al proceso de la enseñanzaaprendizaje de la L2. Así mismo, Mutema y Mariko (2012) concuerdan con lo precedentemente planteado, añadiendo: “The study of errors in students' written texts enables the teacher/lecturer to prescribe the most suitable corrective measures so that the learner reaches a higher level of competence in the L2 which in turn will improve performance." (p. 235).

\section{Método}

\subsection{Diseño de investigación}

Para llevar a cabo la investigación sobre la que se da cuenta en este artículo, se plantearon las etapas que se describen a continuación.

\subsubsection{Selección de la muestra}

En lo que refiere a la muestra, esta es de tipo no probabilístico o elegido y por conveniencia. Lo que definen Hernández, Fernández, y Baptista (2006, p. 241) como el "Subgrupo de la población en la que la elección de los elementos no depende de la probabilidad sino de las características de la investigación". Por lo tanto, la muestra corresponde a estudiantes de pedagogía en inglés de primer, segundo, tercer y cuarto año de formación de una universidad chilena acreditada, constituyendo un total de 72 estudiantes (21 hombres y 51 mujeres), 18 de cada nivel. 


\subsubsection{Instrumento}

Para poder recopilar los datos se confeccionó una actividad de escritura (writing task) en la que se les solicitó a los participantes la producción de discurso escrito de no menos de 250 palabras, respondiendo a lo siguiente: Please refer to why you are studying in this program. Is it because you want to learn the language, you like the teaching profession, or both? What has been your experience thus far?

Para salvaguardar los aspectos éticos de la investigación, se les solicitó a los sujetos que firmaran un consentimiento informado. La aplicación del instrumento se dio a conveniencia del horario de los cursos de los estudiantes, lo que significó utilizar aproximadamente 45 minutos de una de sus clases.

\subsubsection{Proceso de Análisis}

Una vez aplicado el instrumento, se procedió al análisis de los escritos. Este análisis fue realizado por un equipo de investigadores que contaba con un experto en gramática inglesa. Se leyeron todos y cada uno de los escritos y se fueron detectando los errores que aparecían, los cuales fueron tipificados y cuantificados. Para cumplir con el objetivo de la investigación, solo se identificaron errores de carácter gramatical. Se confeccionó una tabla donde se expone el tipo de error y la cantidad de errores cometidos por los informantes.

\section{Resultados y discusión}

Se presentan a continuación los resultados del estudio y su discusión.

\subsection{Resultados por grupos}

En primer lugar, se reportan los estadísticos descriptivos con el fin de caracterizar cada uno de los cuatro grupos, correspondientes a los cursos de interés. Posteriormente, mediante una prueba de diferencia de medias, se procede a identificar diferencias entre los grupos.

\subsubsection{Estadísticos descriptivos}

La Tabla 1 resume los estadísticos descriptivos de la cantidad de errores en cada uno de los niveles. 
Tabla 1. Estadísticos descriptivos para número de errores totales y por nivel.

\begin{tabular}{|l|l|l|l|l|}
\hline & Mínimo & Máximo & Media & Suma \\
\hline Primer año & 0 & 26 & 12,28 & 221 \\
\hline Segundo año & 2 & 16 & 9,89 & 178 \\
\hline Tercer año & 0 & 19 & 7,61 & 137 \\
\hline Cuarto año & o & 9 & 4,72 & 85 \\
\hline
\end{tabular}

$\mathrm{n}=72$

Se aprecia que la media de errores cometidos en cada curso es más alta en primer año y más baja en segundo año. Por su parte la suma de errores totales, esto es, la cantidad de errores totales cometidos por todos los informantes de los cuatro niveles, igualmente tiende a ser menor en los cursos más avanzados.

\subsubsection{Prueba de diferencia de medias}

Se utilizó un análisis de la varianza univariado con el fin de identificar diferencias significativas entre los grupos.

Se encontró que al menos uno de los grupos es significativamente distinto de los demás $(F(3,68)=8.368, \mathrm{p}=.000)$. Para conocer qué pares de grupos son diferentes se observaron las pruebas post hoc, específicamente la comparación de Tukey, debido a que no se violó el supuesto que las varianzas sean iguales $(\mathrm{W}(3,68)=2.035 ; \mathrm{p}=.117)$. La tabla 2 resume las comparaciones post hoc mediante la prueba de Tukey.

Se puede observar que primer año fue significativamente superior que tercer y cuarto año. No hubo diferencias con el grupo de segundo año. Por su parte, cuarto año fue significativamente más bajo que primer y segundo año, pero no que tercero. Esto quiere decir que los extremos (primero y cuarto) fueron diferentes entre ellos, es decir, las personas de cuarto año cometieron significativamente menos errores que las de primero. Los cursos centrales (segundo y tercero) no fueron diferentes. La cantidad de errores que cometieron los estudiantes de tercero no fue significativamente menor que segundo. 
Tabla 2. Pruebas Post hoc.

\begin{tabular}{|c|c|c|c|c|c|}
\hline & & \multirow{2}{*}{$\begin{array}{l}\text { Diferencia } \\
\text { de medias }\end{array}$} & \multirow{2}{*}{ p-valor } & \multicolumn{2}{|c|}{$\begin{array}{l}\text { Intervalo de confianza } \\
\text { de la diferencia al } 95 \%\end{array}$} \\
\hline & & & & $\begin{array}{l}\text { Límite } \\
\text { inferior }\end{array}$ & $\begin{array}{l}\text { Límite } \\
\text { superior }\end{array}$ \\
\hline \multirow[t]{3}{*}{ Primer año } & Segundo año & 2.39 & .43 & -1.76 & 6.54 \\
\hline & Tercer año & $4.67^{*}$ & .02 & .51 & 8.82 \\
\hline & Cuarto año & $7.56^{*}$ & .00 & 3.40 & 11.71 \\
\hline \multirow[t]{3}{*}{ Segundo año } & Primer año & -2.39 & .43 & -6.54 & 1.76 \\
\hline & Tercer año & 2.28 & .48 & -1.87 & 6.43 \\
\hline & Cuarto año & $5.17^{*}$ & .01 & 1.01 & 9.32 \\
\hline \multirow[t]{3}{*}{ Tercer año } & Primer año & $-4.67^{*}$ & .02 & -8.82 & -.51 \\
\hline & Segundo año & -2.28 & .48 & -6.43 & 1.87 \\
\hline & Cuarto año & 2.89 & .27 & -1.26 & 7.04 \\
\hline \multirow[t]{3}{*}{ Cuarto año } & Primer año & $-7.56^{*}$ & .00 & -11.71 & -3.40 \\
\hline & Segundo año & $-5.17^{*}$ & .01 & -9.32 & -1.01 \\
\hline & Tercer año & -2.89 & .27 & -7.04 & 1.26 \\
\hline
\end{tabular}

${ }^{*} \mathrm{p}<.05$

Con esto se puede argumentar que la cantidad de errores que cometen los estudiantes de un curso es significativamente distinta no con cursos inmediatamente sucesivos, sino con cursos más distantes (por ejemplo, primer año no fue diferente de segundo, pero sí de tercero y cuarto).

Tipificación y cuantificación de errores

Los errores identificados dan cuenta de 86 tipos de errores gramaticales, de los cuales los de mayor frecuencia son el uso incorrecto de las proposiciones y calcos sintácticos, los que corresponden a un $20 \%$ del total, tal como se aprecia en la Tabla 3.

Tabla 3. Tipo y cantidad de errores gramaticales.

\begin{tabular}{|c|l|c|c|c|c|c|}
\hline$N^{\circ}$ & \multicolumn{1}{|c|}{ Tipos de Errores } & $1^{\circ}$ & $2^{\circ}$ & $3^{\circ}$ & $\begin{array}{c}4^{\circ} \\
\text { Total de } \\
\text { Errores }\end{array}$ \\
\hline & & & & & & \multirow{2}{*}{67} \\
\hline 1 & Uso incorrecto de preposición & 17 & 20 & 16 & 14 & $\mathbf{6 7}$ \\
\hline 2 & Calco sintáctico & 24 & 15 & 12 & 6 & 57 \\
\hline 3 & Error de forma verbal impersonal & 12 & 14 & 14 & 4 & 44 \\
\hline 4 & $\begin{array}{l}\text { Error de tiempo verbal (presente simple - pasado } \\
\text { simple) }\end{array}$ & 8 & 18 & 11 & 2 & 39 \\
\hline
\end{tabular}




\begin{tabular}{|c|c|c|c|c|c|c|}
\hline 5 & Uso incorrecto de forma verbal en conjugación & 6 & 5 & 8 & 7 & 26 \\
\hline 6 & Omisión de morfema plural & 13 & 2 & 4 & 5 & 24 \\
\hline 7 & Omisión de preposición & 9 & 4 & 6 & 4 & 23 \\
\hline 8 & Uso innecesario de preposición & 6 & 6 & 7 & 3 & 22 \\
\hline 9 & Omisión de la partícula "to" de infinitivo & 16 & 3 & 2 & 1 & 22 \\
\hline 10 & Omisión de pronombre del sujeto "it" & 10 & 5 & 5 & 1 & 21 \\
\hline 11 & Error de orden sintáctico & 8 & 7 & 2 & 1 & 18 \\
\hline 12 & Uso innecesario de pronombre sujeto "it" & 1 & 7 & 1 & 1 & 10 \\
\hline 13 & Uso innecesario de artículo definido & 3 & 5 & 1 & 1 & 10 \\
\hline 14 & Uso incorrecto de determinante & 8 & 0 & o & 2 & 10 \\
\hline 15 & Omisión pronombre de sujeto & 1 & 3 & 3 & 2 & 9 \\
\hline 16 & Uso innecesario de la partícula "to" & 5 & 0 & 3 & 1 & 9 \\
\hline 17 & Uso incorrecto de pronombre relativo & 2 & 2 & 3 & 1 & 8 \\
\hline 18 & Uso innecesario de morfema plural & 1 & 2 & 1 & 4 & 8 \\
\hline 19 & Uso incorrecto de verbo modal & 4 & 1 & 2 & 1 & 8 \\
\hline 20 & Omisión de artículo indefinido & 3 & 3 & o & 1 & 7 \\
\hline 21 & $\begin{array}{l}\text { Error de tiempo verbal (pasado simple - pasado } \\
\text { perfecto) }\end{array}$ & o & 5 & o & 1 & 6 \\
\hline 22 & Error morfológico & 2 & 3 & 1 & o & 6 \\
\hline 23 & Uso incorrecto de adjetivo indefinido & 1 & 2 & 2 & 1 & 6 \\
\hline 24 & Uso incorrecto de conjunción & 3 & 1 & o & 2 & 6 \\
\hline 25 & $\begin{array}{l}\text { Error de tiempo verbal (pasado perfecto - pre- } \\
\text { sente perfecto) }\end{array}$ & 1 & 1 & 4 & o & 6 \\
\hline 26 & $\begin{array}{l}\text { Error de tiempo verbal (pasado simple - presente } \\
\text { simple) }\end{array}$ & 4 & 1 & 1 & o & 6 \\
\hline 27 & Omisión de artículo definido & 1 & o & 3 & 2 & 6 \\
\hline 28 & Omisión de verbo & 2 & 2 & 1 & o & 5 \\
\hline 29 & Uso incorrecto de adjetivo demostrativo & 2 & 1 & 2 & o & 5 \\
\hline 30 & Uso incorrecto de pronombre indefinido & 1 & 1 & 1 & 2 & 5 \\
\hline 31 & Error morfológico de verbo & 3 & 1 & 1 & o & 5 \\
\hline 32 & $\begin{array}{l}\text { Error de tiempo verbal (pasado simple - presente } \\
\text { perfecto) }\end{array}$ & o & 1 & 3 & 1 & 5 \\
\hline 33 & Omisión de pronombre de objeto directo & 0 & o & 2 & 3 & 5 \\
\hline 34 & Omisión de conjunción & 4 & 0 & 1 & o & 5 \\
\hline 35 & Uso innecesario de verbo auxiliar & 0 & 2 & 2 & o & 4 \\
\hline 36 & Uso incorrecto de pronombre de sujeto "it" & 1 & 1 & 2 & o & 4 \\
\hline 37 & Omisión de adverbio & o & 1 & 1 & 2 & 4 \\
\hline 38 & Uso incorrecto de pronombre reflejo & 3 & o & o & 1 & 4 \\
\hline 39 & Uso incorrecto de artículo definido & 1 & 2 & 0 & 0 & 3 \\
\hline 40 & Uso incorrecto de morfema plural & 2 & 1 & 0 & 0 & 3 \\
\hline 41 & Uso incorrecto de verbo & 2 & 1 & 0 & 0 & 3 \\
\hline
\end{tabular}




\begin{tabular}{|c|c|c|c|c|c|c|}
\hline 42 & Uso incorrecto de verbo auxiliar & 2 & 1 & 0 & o & 3 \\
\hline 43 & Uso innecesario de verbo & 1 & 1 & 1 & 0 & 3 \\
\hline 44 & Uso incorrecto de adverbio & 2 & 0 & 1 & 0 & 3 \\
\hline 45 & Uso incorrecto de conector & 1 & 0 & 1 & 1 & 3 \\
\hline 46 & Omisión de sustantivo & 3 & 0 & 0 & 0 & 3 \\
\hline 47 & Uso incorrecto de pronombre personal & o & 2 & o & o & 2 \\
\hline 48 & Uso incorrecto de artículo indefinido & 1 & 1 & 0 & 0 & 2 \\
\hline 49 & Uso innecesario de sustantivo & 1 & 1 & 0 & 0 & 2 \\
\hline 50 & $\begin{array}{l}\text { Error de tiempo verbal (presente simple - presen- } \\
\text { te perfecto) }\end{array}$ & 1 & 1 & 0 & 0 & 2 \\
\hline 51 & $\begin{array}{l}\text { Error de tiempo verbal (pasado perfecto - pasado } \\
\text { simple) }\end{array}$ & o & 1 & 1 & 0 & 2 \\
\hline 52 & Omisión de apóstrofe & o & 1 & 0 & 1 & 2 \\
\hline 53 & Omisión de apóstrofe clítico & 1 & 1 & o & o & 2 \\
\hline 54 & Omisión de verbo auxiliar & 1 & 1 & o & o & 2 \\
\hline 55 & Uso innecesario de conector & 0 & 0 & 2 & 0 & 2 \\
\hline 56 & Uso innecesario de artículo indefinido & 2 & 0 & 0 & 0 & 2 \\
\hline 57 & Uso de doble sujeto & 2 & 0 & 0 & o & 2 \\
\hline 58 & $\begin{array}{l}\text { Error de tiempo verbal (presente simple - pasado } \\
\text { perfecto) }\end{array}$ & 1 & o & 1 & 0 & 2 \\
\hline 59 & $\begin{array}{l}\text { Error de tiempo verbal (partic. pasado - partici- } \\
\text { pio presente) }\end{array}$ & 2 & o & 0 & 0 & 2 \\
\hline 60 & Uso incorrecto de partícula "to" & o & 1 & 0 & 0 & $\mathbf{1}$ \\
\hline 61 & Uso incorrecto de comparativo & o & 1 & 0 & 0 & 1 \\
\hline 62 & Uso incorrecto de adjetivo & o & 1 & 0 & 0 & 1 \\
\hline 63 & Uso incorrecto de participio & o & 1 & 0 & 0 & 1 \\
\hline 64 & Uso incorrecto de pronombre definido & o & 1 & o & o & 1 \\
\hline 65 & $\begin{array}{l}\text { Error de tiempo verbal (presente simple - poten- } \\
\text { cial simple) }\end{array}$ & 0 & 1 & 0 & 0 & 1 \\
\hline 66 & $\begin{array}{l}\text { Error de tiempo verbal (presente simple - condi- } \\
\text { cional simple) }\end{array}$ & o & 1 & 0 & 0 & 1 \\
\hline 67 & $\begin{array}{l}\text { Error de tiempo verbal (presente perfecto - pre- } \\
\text { sente simple) }\end{array}$ & 0 & 1 & 0 & 0 & 1 \\
\hline 68 & Error de tiempo verbal (pasado simple - futuro) & o & 1 & 0 & 0 & 1 \\
\hline 69 & $\begin{array}{l}\text { Error de tiempo verbal (pasado perfecto progre- } \\
\text { sivo - pasado perfecto) }\end{array}$ & 0 & 1 & 0 & 0 & 1 \\
\hline 70 & $\begin{array}{l}\text { Error de tiempo verbal (condicional simple - } \\
\text { condicional perfecto) }\end{array}$ & o & 1 & 0 & 0 & 1 \\
\hline 71 & Omisión de adjetivo posesivo & o & 1 & 0 & 0 & $\mathbf{1}$ \\
\hline 72 & Uso incorrecto de condicional & 1 & 0 & 0 & 0 & 1 \\
\hline 73 & Uso incorrecto de singular & 1 & 0 & 0 & 0 & 1 \\
\hline 74 & Uso incorrecto de adjetivo posesivo & 1 & 0 & 0 & o & 1 \\
\hline
\end{tabular}




\begin{tabular}{|c|l|c|c|c|c|c|}
\hline 75 & Uso incorrecto del pronombre posesivo & 1 & 0 & 0 & 0 & $\mathbf{1}$ \\
\hline 76 & Uso incorrecto del pronombre de objeto directo & 0 & 0 & 0 & 1 & $\mathbf{1}$ \\
\hline 77 & Uso incorrecto de sustantivo & 0 & 0 & 1 & 0 & $\mathbf{1}$ \\
\hline 78 & Uso innecesario de condicional & 1 & 0 & 0 & 0 & $\mathbf{1}$ \\
\hline 79 & Uso innecesario de adverbio relativo & 0 & 0 & 1 & 0 & $\mathbf{1}$ \\
\hline 80 & Uso innecesario de adjetivo comparativo & 1 & 0 & 0 & 0 & $\mathbf{1}$ \\
\hline 81 & Error morfológico de pronombre indefinido & 1 & 0 & 0 & 0 & $\mathbf{1}$ \\
\hline 82 & $\begin{array}{l}\text { Error de tiempo verbal (pasado simple - presente } \\
\text { progres.) }\end{array}$ & 0 & 0 & 0 & 1 & $\mathbf{1}$ \\
\hline 83 & $\begin{array}{l}\text { Error de conjugación de verbo después de auxi- } \\
\text { liar o preposición }\end{array}$ & 1 & 0 & 0 & 0 & $\mathbf{1}$ \\
\hline 84 & Omisión del pseudosujeto & 1 & 0 & 0 & 0 & $\mathbf{1}$ \\
\hline 85 & Omisión del determinante & 0 & 0 & 0 & 1 & $\mathbf{1}$ \\
\hline 86 & Omisión de pronombre relativo & 1 & 0 & 0 & 0 & $\mathbf{1}$ \\
\hline
\end{tabular}

\subsection{Errores de mayor aparición}

A continuación, se presentan los errores más frecuentes con ejemplos auténticos, es decir, sin modificaciones o enmiendas, encontrados en la producción de discurso escrito por parte de los sujetos que constituyen la muestra.

\subsubsection{Errores de preposición}

De acuerdo con nuestra recopilación de datos, el uso incorrecto de preposición es el error más cometido por los sujetos de la muestra, con un total de 67 errores, correspondiendo a un 10,9\%. Algunos ejemplos son:

a) Be on a classroom. (in)

b) Decision that were made in a strike. (during)

c) Teachers form people since a young age. (from)

d) I never had that interest on it. (in)

e) My English has improved widely in comparison of what it was. (to)

Varios de estos errores parecen ocurrir, en gran medida, debido a la influencia de la L1, ya que el sujeto ocupa en muchos casos la preposición más similar o directa a la que usaría en su L1, como es el caso de por ejemplo in-en, of-de, with-con, entre muchos otros, tal como se observa en los siguientes ejemplos:

a) Thinking in another thing. (of) (del español 'pensar en')

b) I have been learning English from years. (for) (del español 'desde años') 
c) Pedagogy is related with the experiences. (to) (del español 'relacionado con')

d) I have always been good with the language. (at) (del español 'ser bueno con')

\subsubsection{Calcos sintácticos}

En segundo lugar, el error más cometido es el calco sintáctico con 57 errores en total, lo que corresponden a un porcentaje de 9,3. Algunos de los errores encontrados se matizan con calcos semánticos.

Este tipo de error ocurre debido a la influencia de la L1, ya que los aprendientes calcan el orden sintáctico propio de su L1 en la producción escrita de la L2. Con esto, nos referimos a que los estudiantes recurren a la estructura oracional del español en una traducción directa al inglés, tal como se presenta subrayado en los ejemplos que siguen:

a) Knowing a little bit of English and discovering something new, supports my choice that I took.

(Conociendo un poquito de inglés y descubriendo algo nuevo, apoya mi decisión que yo tomé)

b) ... both thing are the answer for my decition.

(Ambas cosas son la respuesta para mi decisión)

c) Ideas about how is teaching in the Chilean society.

(Ideas sobre cómo es enseñar en la sociedad chilena)

d) I used to like a lot English when I was at school.

(Me solía gustar mucho inglés cuando yo estaba en el colegio)

e) Thanks to the practicums and techo we here had the chance to meet the other reality of the camps.

(Gracias a las prácticas y techo nosotros aquí tuvimos la chance de conocer la otra realidad de los campamentos)

f) I wanted to study English and be able to talk very well English.

(Yo quería estudiar inglés y ser capaz de hablar muy bien inglés)

\subsubsection{Error de forma verbal impersonal}

Aparecen en tercer lugar errores de la forma verbal impersonal (infinitivo, gerundio y participios presente y pasado) con 44 errores en total, que corresponden a un porcentaje de 7,18. Algunos ejemplos son: 

a) All the problems mention before. (mentioned)
b) I don't regret study this program. (studying)
c) I would get use to go. (used - going)
d) I think work with kids is special. (working)
e) I'm focuse on that. (focused)

\subsubsection{Error de tiempo verbal}

En cuarto lugar, se encuentra el error de tiempo verbal de presente simple en vez de pasado simple, con 39 errores en total, lo que corresponde a un 6,37\%. A modo de ejemplo:

a) I already know I wanted to be a teacher. (knew)

b) My classmate ask me what I want to study. (asked) (wanted)

c) I thought teaching is the most important profession. (was)

d) When I grow up I had to decide. (grew)

e) I start this program. (started)

\subsubsection{Error de forma verbal en conjugación}

El error de forma verbal en conjugación aparece en quinto lugar con un total de 26 errores, lo que corresponde a un 4,24\%. En este caso, el tiempo verbal es correcto, sin embargo, la morfología es inadecuada considerando principalmente el sujeto de la oración. Ejemplos:

a) This program have helped me. (has helped)

b) The teacher evaluate in a different way. (evaluates)

c) The program have changed my mind. (has changed)

d) My experience with this program have been. (has been)

\subsubsection{Omisión del morfema plural -s}

En sexto lugar se aprecia la omisión de morfema plural -s, con 24 errores en total lo que corresponde a un 3,92\%. Ejemplos:

a) I was one of the best student. (students)

b) In terms of social relationship.(relationships)

c) English was one of my favorite subject. (subjects)

d) Also because I want to change the live of my future student. (lives) (students) 
e) I've been working on different type of schools. (types)

\subsubsection{Omisión de preposición}

El séptimo error más cometido es la omisión de preposición con 23 errores cometidos, lo que corresponde a un porcentaje de 3,75 del total. Esto se puede deber a la interferencia de la L1, puesto que, en los ejemplos citados, en español no se requiere de preposición. Ejemplos:

a) I remember that I liked to listen__ music in English a lot. (to)

b) I think is good that we have native speakers teacher. (as)

c) Even if just one _ _ of the two hundred thousand student learn something. (out)

\subsubsection{Uso innecesario de preposición}

El octavo error más cometido es el uso innecesario de preposición con 22 errores, lo que corresponde a un porcentaje de 3,59\%. Este error se presenta principalmente por la influencia de la L1, ya que, a diferencia del punto anterior, en español efectivamente se utilizan ciertas preposiciones de acuerdo al contexto. Ejemplos:

a) I trust in my friend because he gave me advice for my first practicum.

b) Also, when I helped to my friend, I liked the feeling.

c) I'm in my third year now and don't regret about anything.

\subsubsection{Omisión de la partícula "to" de infinitivo}

El noveno error más cometido es la omisión de la partícula "to" en infinitivo con 22 errores en total, lo que corresponde a un porcentaje de 3,59. Se puede sostener que este tipo de error es causado por la influencia de la L1, ya que el infinitivo en español no posee esta característica. Ejemplos:

a) It's challenging __ study. (to)

b) I would like _ travel. (to)

c) I need __ learn very well. (to)

d) It is wonderful _ _ teach content and interact with the students. (to)

\subsubsection{Omisión pronombre sujeto "it"}

En décimo lugar aparece el error de omisión de pronombre sujeto "it" con un 3,43\%. Al igual que el error anterior, este se debe influencia de la L1, 
ya que en español la declinación verbal revela al sujeto y, por lo tanto, el pronombre sujeto puede ser omitido: Esto no ocurre en inglés. Ejemplos:

a) I'm into the teaching profession as _ is expected from my major.

b) I think that _ is one of the best majors.

c) __ is one of the things that I can do right and I can be successful.

d) I didn't know how __ could be.

\section{Discusión y conclusiones}

A partir de los resultados obtenidos se observaron un total de 621 errores, de entre los cuales de detectaron ochenta y seis tipos cometidos por los 72 participantes que constituyen la muestra. Los resultados verifican efectivamente que los sujetos de los niveles más altos cometen menos errores que los de los niveles más bajos. Se evidencia que la mayor diferencia está entre los sujetos de primer y cuarto año.

En cuanto al tipo de errores cometidos por los participantes, un fenómeno interesante ocurre con los calcos sintácticos, omisión de la partícula "to" y error de forma verbal impersonal, los cuales disminuyen significativamente dependiendo del nivel que los sujetos estén cursando en la carrera. No obstante lo anterior, los otros tipos de errores muestran comportamiento dispar, como el error de conjugación (presente simple en vez de pasado simple), donde los sujetos de segundo y tercer año presentan más errores que los sujetos de primero o cuarto año.

Cabe resaltar que las preposiciones presentan una alta dificultad para los sujetos de la muestra, siendo el error más frecuente el mal uso, el uso innecesario y la omisión de las mismas. Estos hallazgos son coincidentes con los de Khansir y Ilkhani (2016) quienes obtuvieron resultados similares en su estudio de errores gramaticales cometidos por estudiantes universitarios Iraníes. Allí se presenta el error de preposición como el error más cometido.

Asimismo, el segundo error más cometido por los sujetos de nuestra muestra fue el calco sintáctico. Se han encontrado resultados similares en el estudio realizado por Zhan (2015) quien analizó las muestras escritas de estudiantes chinos de EFL. Zhan (2015) llamó este tipo de error ChineseEnglish expressions y fue el cuarto error más cometido en su estudio. Zhan (2015), a este respecto, sostiene: “They are so greatly affected by their mother tongue that they often ignore the basic grammatical 
structure of English. Whether in simple and short or complex and long sentences, they may resort to Ll equivalents or Chinese expressions in the word sequence. And in some more complicated sentences students make more errors." (p.77).

En el caso de Mulyono (2016) los resultados de su investigación revelaron que el error sintáctico cometido con mayor frecuencia era el uso de presente simple en vez de pasado simple, error que en el estudio también alcanzó una alta notoriedad, siendo clasificado en el $4^{\circ}$ puesto. Mulyono (2016) sostiene que esto se debe a lo siguiente: "In this case, the student still uses simple present tense verb in their writing. It seems that he/she ignores the rule of grammar in English or he/she has not reached the material yet, so that he/she still uses simple present tense instead of simple past tense form." (p.11).

Por su parte Zhan (2015) encontró que el error más cometido por estudiantes chinos de EFL fue el error de tiempo y forma verbal, los que separó en sub categorías de: tense, 3rd -person singular y past tense, past participle and others. En el estudio reportado aquí, en cambio, los errores fueron separados por tiempo verbal y forma verbal. La forma verbal tiene dos categorías: 'forma verbal impersonal' y 'forma verbal en conjugación'. El error de 3rd person singular de Zhan (2015) es el equivalente de 'error forma verbal en conjugación', que es el quinto error más cometido en el estudio presentado en este artículo.

En cuanto al uso de artículos, Khansir y Shahhoseiny (2013) hallaron que el error más frecuente cometido por estudiantes universitarios de China y Corea del Sur fue la omisión del artículo definido. Esto es debido a la influencia de su L1 puesto que, por un lado, en lengua coreana no existen los artículos indefinidos ni los definidos y en chino los artículos son utilizados como distintos determinantes del sustantivo. En cambio, en este estudio, la omisión del artículo definido ( 7 casos, 1,1\%) fue el vigésimo séptimo error, es decir, tienen poca incidencia, ya que en español y en inglés hay ciertas coincidencias de uso.

En la investigación hecha por Kurniawan (2016) en Indonesia a estudiantes de tercero y cuarto año de colegio y primer año de universidad muestra la comparación de los errores cometidos por cada año. Al igual que en nuestra investigación, se encontró un alto número de omisión del morfema plural -s. Kurniawan (2016) señala que la omisión del morfema -s se debe a confusión y poco conocimiento del idioma y que esta persiste en cada año. Sin embargo, en nuestro 
estudio los sujetos de primer año omiten el morfema plural -s más que los sujetos de los años superiores.

En conclusión, podemos observar en este estudio que el desarrollo de la interlengua se ve marcado por el nivel que cursan los sujetos estudiados, es decir, a mayor nivel de curso de la L2, menor cantidad de errores cometidos en su producción escrita. Cabe destacar que al ser nuestros sujetos de estudio aprendientes en formación para ser profesores de EFL, nos parece preocupante que, al nivel terminal de sus estudios de pregrado, aún persisten ciertos errores, los cuales podrían llegar a presentar una posible fosilización en el futuro. Es por ello que consideramos importante que este estudio sea tanto un aporte para la discusión académica como un precedente para futuros estudios con este mismo tipo de sujetos. Ello podría contribuir a mejorar los procesos de aprendizaje y erradicar los errores en la producción de discurso en la L2.

\section{Referencias bibliográficas}

Ahour, T., \& Mukundan, J. (2012). Errors and variations of TESL students' written description. Pertanika Journal of Social Sciences \& Humanities. 20(1), 55-64.

Bailini, S. (2012). Interacción y desarrollo de la interlengua: E-tándem español-italiano. Tesis doctoral, Universidad de Salamanca.

Barahona, M. (2016). English language teacher education in Chile. Estados Unidos: Routledge.

Bourguignon, I. (2008-2009). Análisis de errores de interlengua de aprendices neelandófonos universitarios del español. Tesis de Máster, Universiteit Gent, Bélgica.

Campillos Llanos, L. (2014). Las preposiciones en el habla no nativa de nivel intermedio: análisis de la interlengua basado en corpus. Revista Nebrija de Lingüística Aplicada, 16, 5-27.

Corder, S. P. (1967). The significance of learners' errors. IRAL, 5(4), 161-170.

_. (1981). Error Analysis and Interlanguage. Oxford: Oxford University Press.

Doquin de Saint Preux, A. \& Sáez Garcerán, P. (2014). Análisis de errores en dos tipos de producciones escritas de alumnos franceses de 
ELE de nivel avanzado. Revista Nebrija de Lingüística Aplicada, 17, 114-128.

Ellis, R. (2012). Language teaching research \& language pedagogy. (1 $\left.{ }^{\mathrm{a}} \mathrm{ed}.\right)$. Oxford: Wiley-Blackwell.

Fernández López, S. (1997). Errores e interlengua en el aprendizaje del español como lengua extranjera. Didáctica, Lengua y Literatura, 7, 203-216.

Hernández, R., Fernández, C., \& Baptista, P. (2006). Metodología de la investigación. ( $4^{\mathrm{a}}$ ed.). México: McGraw Hill.

Heydari, P. \& Bagheri, M. (2012). Error Analysis: Sources of L2 Learners' Errors. Theory and practice in language studies, 2(8), 1583-1589.

Jagusztyn, A. (2014). Attitudes toward ESL use of corpora in second language writing courses and its effects on error-correction identification and learning by L2 learners of English. Tesis de Máster, University of Illinois at Urbana-Champaign, EE.UU.

James, C. (1998). Errors in Language Learning and Use. New York: Routledge.

Johnson, K. (2001). An introduction to foreign language learning and teaching. London: Longman.

Khansir, A. \& Ilkhani, M. (2016). A study of written grammatical errors of Iranian EFL learners at undergraduate level. Theory and Practice in Language Studies, 6(2), 268-273.

Khansir, A., y Shahhoseiny, J. (2013). The study of written errors of EFL preuniversity learners. Journal of Language Teaching and Research, 4(6), 1253-1258.

Krashen, S. (1981). Second Language acquisition and second language learning. Oxford: Pergamon Press.

Krishnamurthy, S., Kangira, J., Tjiramanga, A., \& Beukes, B. (2011). An analysis of English errors made by Polythecnic of Namibia students. NLJC, 5(1), 1-15.

Kurniawan, D. (2016). A comparative study of error analysis in writing narrative texts made by junior, senior and university students. Tesis de Magíster, Universitas Muhammadiyah, Indonesia.

Li, X. (2015). Analysis on lexical errors in writings of Mongolian English majors. Theory and Practice in Language Studies, 5(12), 2565-2570. 
Liu, N. (2012). Análisis de errores y evolución de la interlengua de los estudiantes sinohablantes: esbozo de un estudio longitudinal. Tesis de Magíster, Universidad de Barcelona, España.

Mineduc. (s.f.). En Mineduc. Recuperado de https://www.mineduc.cl/ Mulyono, E. (2016). Errors in narrative text committed by students in grade XI of vocational high school (SMK) State 4 Surakarta academic year 2014/2015. Tesis de Máster, Universitas Muhammadiyah, Indonesia.

Murad, T., \& Kalil, M. (2015). Analysis of errors in English writings committed by Arab first-year college students of EFL in Israel. Journal of Language Teaching and Research, 6(3), 475-481.

Mutema, F., \& Mariko, I. (2012). Common errors in second language (L2) speakers' written texts. A case of first year first semester (L1:S1) Arts students at Midlands State University: An error analysis approach. The Modern Journal of Applied Linguistics, 4(4), 208-235.

Nemser, W. (1971). Approximative systems of foreign language learners. IRAL, 9, 115-130.

Sánchez Jiménez, D. (2010). La adquisición de la lengua extranjera española por estudiantes filipinos: análisis descriptivo y explicativo de la interlengua. redELE, 18, 58-84.

Selinker, L. (1972). Interlanguage. International review of applied linguistics in learning teaching, 10(3), 209-231.

Tarone, E. (2006). Variation in Interlanguage. (4 ed.) London: Edward Arnold.

(2014). Enduring questions from the interlanguage theory. En Han, Z. y Tarone, E. (eds.), Interlanguage forty years later (pp. 7-27). Philadelphia: U.S.A.

Yuanrong, D., y Mingcai, S. (2010). Analyzing interlanguage of non-English majors by proposed semantic criteria. Chinese Journal of Applied Linguistics, 33(2), 48-59.

Zhan, J. (2015). Frequent Errors in Chinese EFL Learners' Topic Based Writings. Canadian Center of Science and Education, 8(5), 7-81.

Zheng, C., y Park, T. (2013). An analysis of errors in English writing made by Chinese and Korean university students. Theory and Practice in Language Studies, 3(8), 1342-1351. 